\title{
A Vehicle Mobility Model of VANET by Considering Avoidance
}

\author{
Deng Yuanyuan, Zhou Peng, Xiao Xiaoqiang \\ College of Computer, Natonal University of Defense Technology, Changsha, China
}

\section{Email address:}

Llqq000@163.com (Deng Yuanyuan),1092873889@qq.com (Zhou Peng),xqxiao@nudt.edu.cn (Xiao Xiaoqiang)

\section{To cite this article:}

Deng Yuanyuan, Zhou Peng, Xiao Xiaoqiang. A Vehicle Mobility Model of VANET by Considering Avoidance. Science Discovery.

Vol. 4, No. 5, 2016, pp. 336-341. doi: 10.11648/j.sd.20160405.24

Received: September 25, 2016; Accepted: November 2, 2016; Published: November 5, 2016

\begin{abstract}
Environment of communication, traffic density and other factors have a significant impact on the signal transmission of Vehicular Ad Hoc Network (VANET). Therefore, a suitable mobility model is of great importance for the design and performance evaluation of VANET protocol. In this paper, we expand the IDM_LC model by considering the real features of vehicle mobility and introduce the avoidant behaviors for special vehicles (e.g. ambulances, fire engines, etc.). We propose a mobility model calls IDM_LCA, and complete the simulation scenarios using a network simulation tool-NS3. The experimental result shows that the mobility model IDM_LCA is effective and feasible.
\end{abstract}

Keywords: VANET, Mobility Model, IDM_LC, IDM_A, NS3

\section{一种考虑避让的VANET车辆移动模型}

邓媛媛, 周鹏, 肖晓强

国防科学技术大学计算机学院, 长沙, 中国

邮箱

11qq000@163. com (邓媛媛)，1092873889@qq. com（周鹏）, xqxiao@nudt. edu.cn（肖晓强）

摘要：由于通信环境、车流密度等因素对车载网络 (VANET) 信号的传输有很大影响, 因此合适的移动模型在车载网路 由协议的设计和性能评估中扮演着重要的角色。本文基于真实的车辆运行特性, 在考虑驾驶员跟驰行为的基础上, 引 入对特殊车辆 (比如急救车、消防车等) 的避让行为, 对车载移动模型IDM_LC进行拓展, 提出了一个考虑避让行为的 移动模型IDM_A, 使其能够有效模拟普通车辆对特殊车辆的避让行为, 并通过网络仿真工具NS3完成仿真场景的搭建。 实验结果验证了所提出的IDM_LCA移动模型的可行性及有效性。

关键词: 车载自组网, 移动模型, IDM_LC, IDM_A, NS3

\section{1. 引言}

车载网络 (Vehicular Ad Hoc Network, VANET) 是智 能交通的一个重要组成部分, 它以车辆作为移动自组网的 节点采集发送实时的道路交通信息，不仅能够通过冲撞规 避和安全预警系统改善驾驶员的安全驾驶环境, 还可以减
少交通事故伤亡人数以及缓解交通拥塞 [1]。尽管在真实 环境中评估车载网络的性能非常关键, 但是由于逻辑难度、 经济问题和技术限制, 使得仿真成为验证车载网路由算法 的最好选择。当设计仿真环境时, 必须定义合适的车辆移 动模型才能恰当的反映真实的车辆运动模式。 
早期的VANET仿真环境通常采用随机移动模型, 例如 随机漫步模型（Random Walk Mobility Model）、随机路 点移动模型 (Random Waypoint Mobility Mode1)、随机 方向移动模型 (Random Direction Mobility Mode1) [2]。 但车辆行驶轨迹受交通环境和法规环境等客观因素的影 响, 并非随机移动, 所以随机移动模型难以准确模拟VANET 的场景。随着人们对车载网研究的不断深入, VANET研究 者根据不同的研究方向提出了不同的模型, 车辆移动模型 的形式和复杂性都得到了极大的扩充。例如根据相邻车辆 信息建立车辆跟驰行为的IDM模型, 可以进行十字路口管 理和多车道控制的 IDM_IM移动模型, 可以切换车道的 IDM_LC移动模型等 [3], 但是它们大都基于单一种类车辆 的标准驾驶行为建立, 没有考虑道路场景中特殊车辆的加 入而引起的车辆避让行为 (图1)。这种避让行为将打破 车流的基本行驶模式, 影响路由协议的性能评测。

本文基于真实的车辆运行特性, 在考虑驾驶员跟驰行 为的基础上, 对车载网络移动模型IDM_LC (Intelligent Driver Model with Lane Change) 进行拓展, 提出了一 个考虑避让行为的IDM_A (Intelligent Driver Mode1 with Avoidance) 模型, 通过网络仿真工具NS-3完成 VANET场景搭建, 并采用离散参数取值的方法对不同车流 密度下的车辆避让行为所引起的车辆轨迹改变进行仿真 分析。仿真结果表明, 所搭建的场景能够有效模拟车辆的 避让行为, 不同车流密度下车辆行驶轨迹受避让信号的影 响不一。

\section{2. 考虑避让行为的车辆移动模型IDM_A}

普通车辆对特殊车辆的避让行为, 并不完全符合基本 的变道驾驶模式。如图1所示, 当特殊车辆 (救护车) 前 行时, 其前方的车辆将变道驶入两侧的车道。在车载网络 路由算法中, 这种行车模式的改变对车辆的行驶方向预判、 车流密度以及连同性的估算等方面产生重要影响, 所以能 反映车辆交通真实行为的移动模型尤为关键。

鉴于IDM_LC (Intelligent Driver Model with Lane Change ） [4]模型对车辆的基本行驶模式的良好体现, IDM_A移动模型在IDM_LC模型的基础上进行拓展, 通过进 行车辆分类以及建立通信来模拟车辆对避让信号的接收 等方式, 以实现车辆避让驾驶行为的模拟。

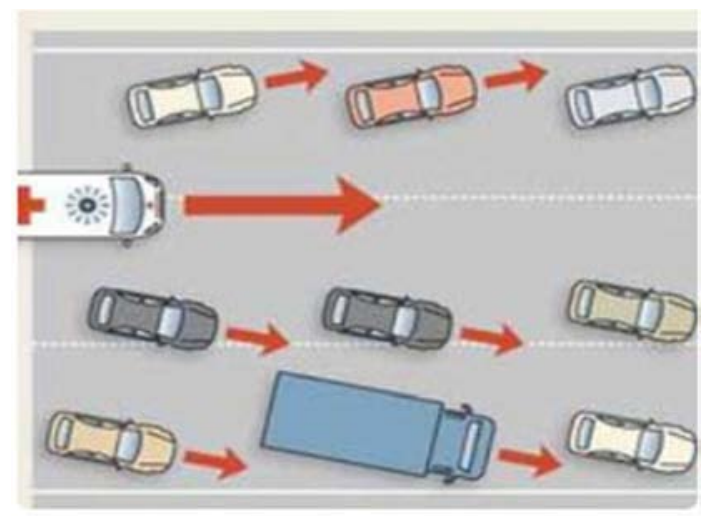

图1 车辆避让行为。

\section{1. IDM_LC模型}

IDM LC 模型是在IDM (Intelligent Driver Mode1) 模型的基础上发展而来。IDM模型考虑驾驶员的跟驰行为, 车辆的速度受前车速度的影响。IDM_LC使用MOBIL模型控 制车辆的变道行为。

当下面的不等式成立时, 则允许进行变道:

$$
\begin{gathered}
a^{l}-a \pm a_{\text {bias }}>p\left(a_{\text {new }}-a_{\text {new }}^{l}\right)+a_{\text {thr }} \\
a_{\text {new }}^{l}>-a_{\text {safe }}
\end{gathered}
$$

在不等式 (1) 中, $a^{l}-a$ 表示当前车辆选择变道到 $l$ 之 后的加速度增加的量, $a_{\text {new }}-a_{\text {new }}^{l}$ 表示当前车辆进入的 候选车道后, 后面的那辆车加速度的损失, $p$ 为礼貌参数, 当 $p$ 的值在 0 到 1 之间，司机的行为越礼貌，则 $p$ 的值越小。 $a_{t h r}$ 表示允许车道改变的最小加速度增益阈值, 如果它的 值很大, 那么该车辆将不会进行变道。 $a_{\text {bias }}$ 用于调整模型 中偏好的移动行为, 在IDM_LC中车辆变换车道会引入 $a_{\text {bias }}$, 使模型与车辆右侧通行的行为相符合。不等式 (2) 表示 在当前车辆变换到 $l$ 车道以后, 在新的车道上, 当前车辆 后面的车的减速度必须大于安全值 $a_{s a f e}$ 。

IDM_LC移动模型虽然可以良好的反映车辆的超车变 道行为, 但是无法对车辆的避让变道行为进行有效的模拟。 主要原因有以下两点:

第一、IDM_LC移动模型与其他大多数移动模型一样, 设定所有的车辆节点都处于平等的状态, 且遵 守同样的移动规则, 并不对车辆进行优先级别 的区分。

第二、IDM_LC移动模型只是以当前车辆为中心, 通过 收集其附近车辆的行驶状态信息（比如距离、 位置、速度、加速度等) 模仿自然驾驶行为, 并没有考虑远距离车辆之间的信息交互。所以, 仅能有效反映驾驶员对视野中的交通状况做 出的反馈行驶行为, 无法对声音 (比如避让信 号, 警笛) 等, 可以较远距离传输的信息做出 恰当的反映。

\subsection{IDM_A模型结构}

针对IDM_LC无法有效反映避让驾驶行为的两大问题, 构建IDM_A车辆移动模型结构如图2所示。车辆分为 $\mathrm{A}$ (特殊) 和 $\mathrm{B}$ (普通) 两种不同类型, 并假定每辆车都可以通过WiFi 接受到特殊车辆发出的避让信号。车辆的行驶轨迹模拟方 式如下:

(1) A类特殊车辆

$A$ 类车辆只在绿色安全车道 (中间车道) 行驶, 其行 驶轨迹按IDM移动模型设定, 车辆不因外界情况进行变道 行为。并间断性广播避让信息, 模拟紧急车辆的警笛信号。

(2) B类普通车辆

当场景中没有 $\mathrm{A}$ 类车辆行驶时, B类车辆按照IDM_LC 车辆移动模型设定的轨迹行驶。一旦通过WiFi信息交换获 得 $\mathrm{A}$ 类车辆的存在, 则在保证安全的情况下进行避让驾驶。 其具体方式为: 中间车道上位于紧急车辆前方的 $B$ 类车辆, 
在保证安全的条件下驶入其他车道; 而非中间车道的B类 车辆进行减速行驶, 以确保中间车道行驶的车辆能够安全 变道。

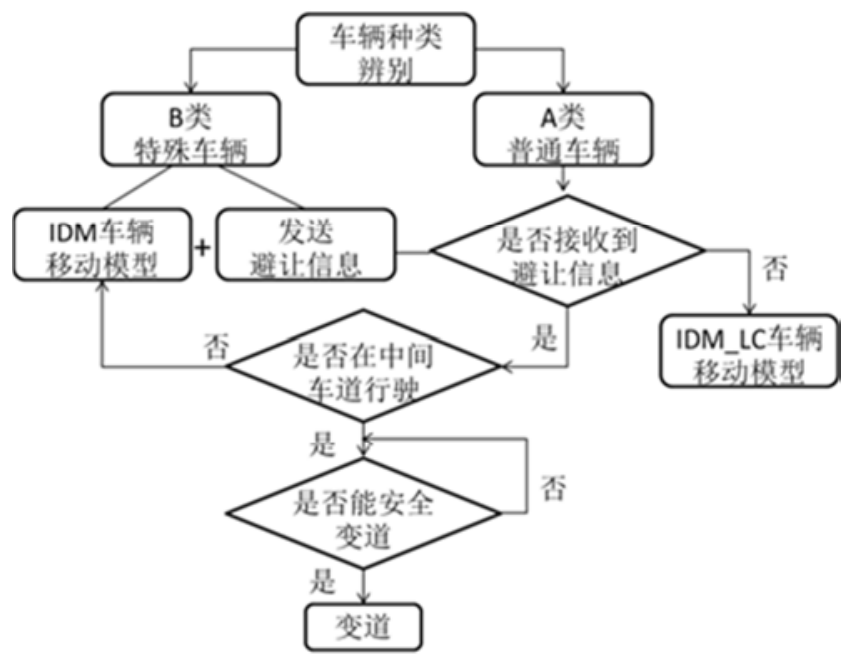

图2 IDM_A模型结构。

\section{VANET避让仿真场景搭建}

由于车载网的特殊性, 对VANET的仿真不仅要考虑网 络相关问题, 也要考虑车辆本身的移动性。针对不同的测 试需求, 根据网络仿真器与移动模型的交互情况, 通常将 VANET仿真划分为三种形式: 孤立方式、嵌入方式、融合 方式 $[5]$ 。

孤立方式允许移动性和网络建模独立发展, 是早期 VANET仿真的主要形式, 但是不适应绝大多数VANET研究对 交互的广泛需求。融合方式是目前主流的仿真形式, 这种 方式可以使最先进的移动模型或交通仿真器与最新且高 效的网络仿真器一起工作。例如TraNS [6], 它将交通仿真 器SUMO [7] 与网络仿真器NS-2 [8] 相融合, 通过接口实现从 SUMO 中提取路径后传给NS-2和命令从NS-2发送至SUMO的 交互。但是由于同时运行两个仿真器, 所需要的计算量非 常大, 且不同仿真器之间存在通信延时。对动态拓扑变化 快的VANET来说并不是理想的选择。嵌入方式使移动模型 和网络仿真器融合在单一的仿真器中, 仿真器之间可以简 单而高效的交互, 使节点位置更为精确的同步。对这种嵌 入方式的仿真结构对交通安全、交通效率等对信息收发的 实时性要求高的VANET相关应用仿真准确性更高, 是NANET 仿真的未来发展方向, 但是这种嵌入方式存在移动仿真或 网络仿真的能力限制的问题一直是有待解决的难题

\section{1. 仿真工具NS-3}

开源网络仿真器NS-3 (Network Simulator 3) 是一 款面向网络系统的, 主要用于研究和教学的离散事件模拟 器 [9]。它在多款主流网络仿真模拟器的基础上研发, 吸 收优点, 摒弃缺陷。且NS-3中包含了 IEEE 802.11p PYY/MAC 层的实现, 因此, NS -3 是对于VANET是最合适的仿真器。

图 3 是NS-3的功能模块示意图。从图中可知NS-3有着 强大的模拟功能。其基本功能模块分为六层: 核心层、网
络层、移动与互联网层、协议与应用等模块层、辅助工具 层及测试层 [10]。NS-3仿真支持基于IP和非IP网络研究, 大多数研究人员关注点主要集中在wireless/IP模拟, 以 及各种静态或动态的路由协议, 比如OLSR、AODA [11]等。 NS-3本身包含一些简单的移动模型, 例如静态模型, 随机 漫步模型等, 其节点的运动模式和大多数VANET所需的车 辆行驶场景相差甚远, 但为VANET实现嵌入式仿真架构奠 定了基础。

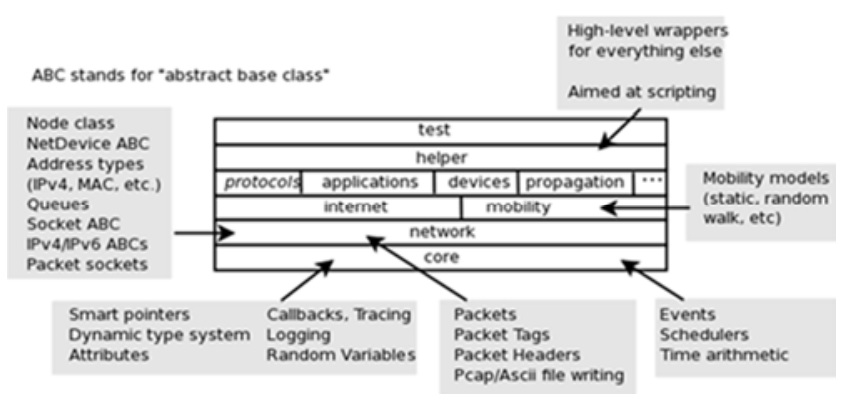

图3 NS-3的功能模块示意图。

3. 2 . 基于 NS-3 的IDM_A移动模型搭建

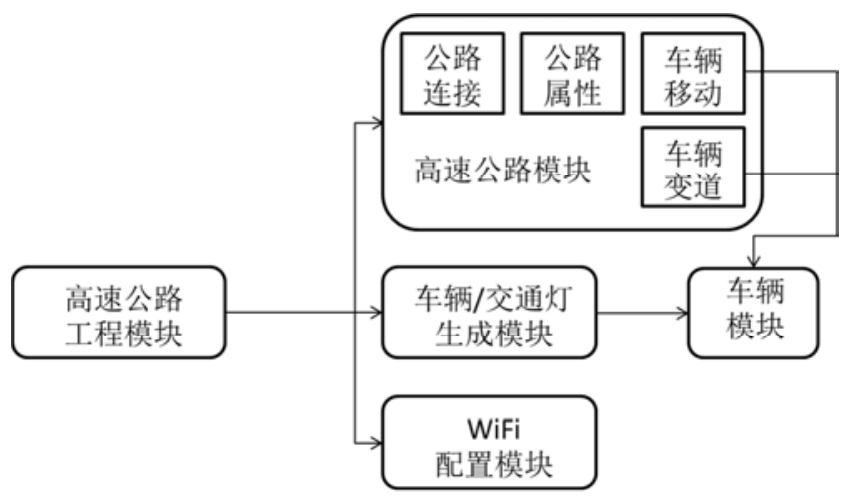

图4 IDM A 移动模型基本架构。

因Bradley Dupont在网络仿真器 NS-3 中完成了以 IDM_LC 移动模型为基础, 添加了道路场景因素的 VANET-Highway [12]场景模块的搭建, 成功将移动模型和 网络仿真器融合在单一的仿真器中, 实现了嵌入式仿真架 构; 对交通安全、交通效率等对信息收发的实时性要求高 的VANET相关应用仿真准确性更高。IDM_A车载网络移动模 型的搭建沿用了车载网仿真场景VANET-Highway基本架构。 在其基础上实现VANET避让仿真场景实现。

具体方法如下:

(1) 将模拟场景由单向单条公路加以拓展。由于只 需要模拟避让行驶行为, 设定为车道宽度为 $5 \mathrm{~m}$, 长度为 $1000 \mathrm{~m}$ 长三车道单向行驶路段。车道坐标 由纵坐标表示, 从上至下分别为 $10.0,5.0,0.0$ 。 中间车道 (纵坐标为 5.0 ) 为紧急车辆绿色通道。

(2) 车辆分为两种类型。B类紧急车辆Vehicle $I D=-1$, Type ID $=3$; A类车辆Type ID $=0$, Vehicle ID根据出现时间从 0 开始以整数赋值。 
（3）实现避让消息的广播与接收, 需在vehicle类中 加入packet成员并编写相关函数代码, 使其具 有简单的消息收发功能。

避让实现的关键处理流程如图5所示。Aside为避让转 道标志位, Aside $=0$ 表示当前车辆不需要进行变道, 比如 当前车辆处于紧急车辆后方, 即使处于中间车道且在信号 传输范围内, 但是不需要进行变道; Aside $=1$ 表示此时的 场景环境需要当前车辆在保证安全的情况下进行变道行 驶。

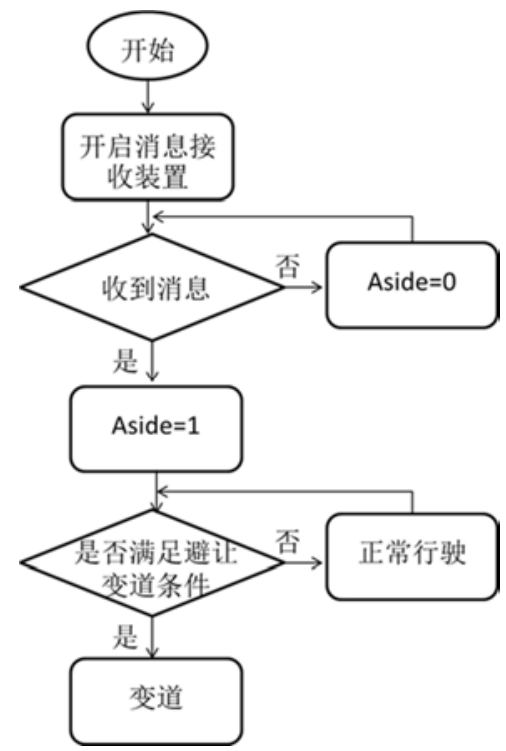

图5 避让变道实现流程。

为了模拟避让驾驶行为, 构建三车道单向行车路段场 景。设置公路长度为 $1000 \mathrm{~m}$, 单条车道宽度为 $5 \mathrm{~m}$, 所有车 辆从左向右行驶。代码通过waf编译运行后, 可以获得记 录车辆移动信息的vehicleTrace. csv文件和记录通讯信 息的networkTrace. csv文件，这两个trace文件用于可视 化处理可视化。可视化软件vehicleviewer使用NetBeans 进行编译处理, 运行结果如图6 所示。

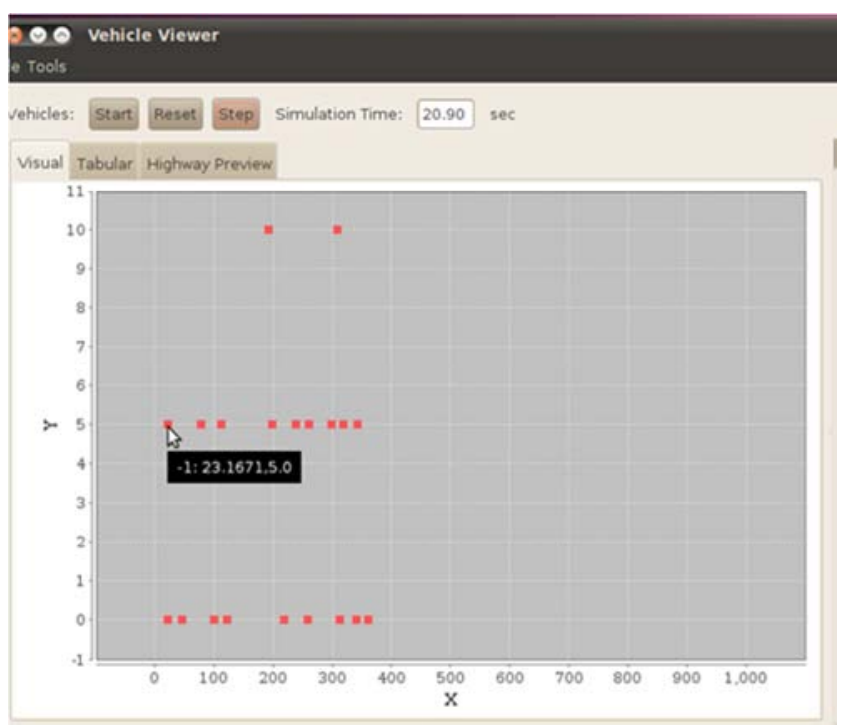

图6 单向三车道场景 (vehicleviewer)。

\section{4. 仿真及结果分析}

\section{1. IDM_A避让仿真}

为了反映车辆在考虑避让情况的行驶状况, 设定紧急 车辆在 $20 \mathrm{~s}$ 时加入场景中间车道, 车流密度 $\mathrm{f} 1 \mathrm{ow}=2$ 辆 $/ \mathrm{s}$, $W i F i$ 通讯距离为 $200 \mathrm{~m}$ 。因每条车道宽度为 $5.0 \mathrm{~m}$, 且方向为 $\pi / 2$ 的方向, 所以在仿真场景中, 3 条车道的坐标由横坐 标标定，其值从左至右分别为 $0.0 、 5.0 、 10.0$ 。

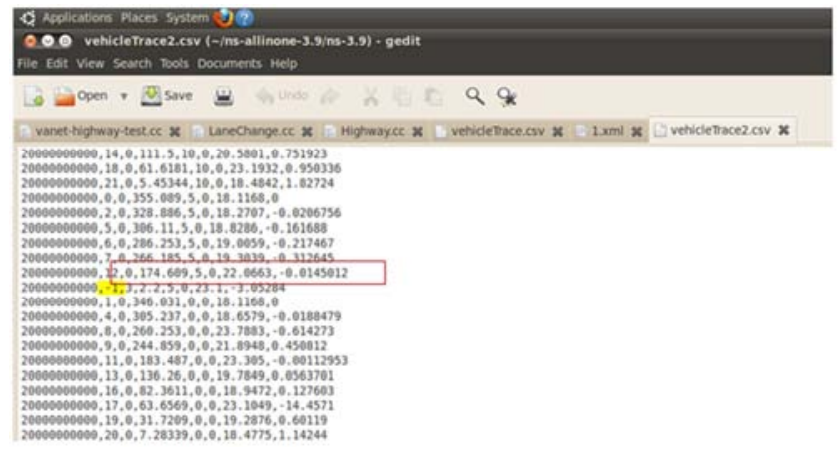

图7 20s 时的车辆分布信息。

图7中, 参数设定从左至右依次为模拟时间 (间隔时间 $=0.1 \mathrm{~s}) 、$ Vehicle ID、Type ID、横坐标 $(\mathrm{m})$ 、纵坐标 $(\mathrm{m})$ 、 速度 $(\mathrm{m} / \mathrm{s})$ 和加速度 $(\mathrm{m} / \mathrm{s} 2)$ 。特殊车辆的 $\mathrm{ID}=-1$, 显然, Vehicle ID $=12$ 的车辆在避让信号通信范围内。在IDM_LC 和IDM_A两种车辆移动模型场景下对Vehicle $\mathrm{ID}=12$ 的车 辆行驶轨迹进行模拟。模拟时间 20-22s之间 (以0. 1 秒为 一个时间节点) 时Vehicle ID $=12$ 的车辆的车道变换如图 4 所示。 $\mathrm{S}$ 表示车道的坐标。设定其坐标为 $\mathrm{S}$ 表示车辆行驶的 每经过10步, 模型会进行车辆变道判定。变道计算发生在 加速和平移计算之前。图8为仿真第 $21 \mathrm{~s}$ 时, 道路上的车辆 分布情况。显然, IDM_A 车辆移动模型场景下的Vehicle $\mathrm{ID}=12$ 的车辆在 21 秒已经成功变道, 完成了避让行驶。

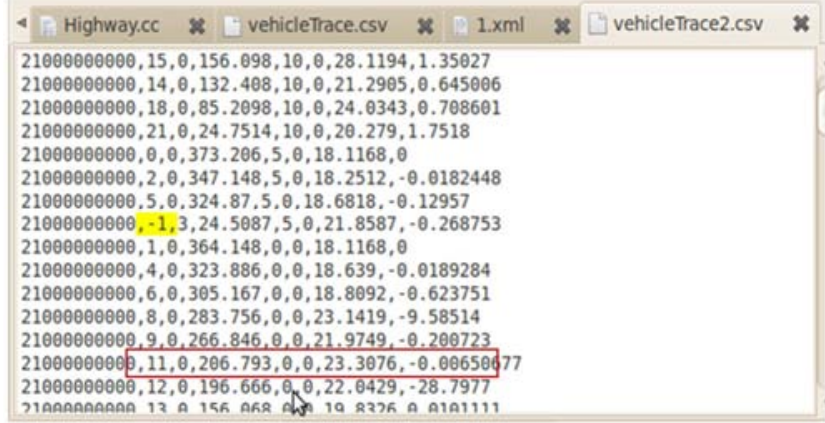

图8 21s时的车辆分布信息。

\subsection{IDM A 模型中车流密度对VANET拓扑结构的影响}

紧急车辆加入车道后对同路段的车辆具有影响这一 点是冊庸置疑的。为了测试IDM A 模型中的避让行驶在不 同车流密度场景中对VANET拓扑结构的影响能力，在 IDM_LC和IDM_A两种模型的 $1000 \mathrm{~m}$ 公路场景中, 分别对车流 密度flow不同取值时, 紧急车辆和普通车辆的行驶状况, 以及避让造成的路由空洞状况进行分析 
加入场景的车辆根据车流密度和车道分布情况随机 选择车道加入。虽然车流密度增加, 但加入中间车道的车 辆数目并不是严格按照车流密度递增的。所以特殊车辆的 行驶时间并没有呈现绝对递增趋势, 但是两种不同的移动 模型场景, 由于其车流密度一样, 在特殊车辆驶入前场景 中的车辆分布一致, 所以可以进行比较分析。

如图9所示, 当紧急车辆驶入场景时, 其前方同车道 的车辆数目及位置分布会对其行驶速度产生影响特别是 在车流密度为3-5时, IDM_A模型场景特殊车辆行驶 $1000 \mathrm{~m}$ 所需的时间明显比IDM_LC模型场景少, 这段时间车辆避让 效果明显。在车流密度特别小时, 因场景中的车辆少, 而 且为了保证行车安全, 即便前面没有其它车辆阻碍, 特殊 车辆的速度只能达到所设定的速度最大值, 所以避让行为 对特殊车辆的行驶影响力度低, 避让带来的效果不明显。 在道路上每加入一辆车前会都会提前判断该车道是否有 其它车辆, 若有, 则只有等最接近道路入口的车辆行驶距 离大于最小安全距离时新生成的车辆才会驶入场景。所以, 即使车流密度增加, 在车流密度大于 10 以后, 紧急车辆在 IDM_LC模型场景与IDM_A模型场景下行驶所需的时间都趋 于一个定值。

紧急车辆加入车道后对同路段的车辆具有影响。中间 车道的车辆因为需要避让特殊车道的原因驶入其他车道, 使其它车道的密度增加, 影响了非中间车道的车辆行驶速 度。因将 0.5 辆 $/ \mathrm{s}$ 作为仿真测试的最小车流密度, 在 $20 \mathrm{~s}$ 紧急车辆驶入时, Vehicle ID $=10$ 的车辆已经存在场景中。 抽取Vehicle ID=10的车辆在不同模拟场景行驶所需的时 间进行统计。如图10所示, 在IDM_A场景中Vehicle ID=10 的车辆所需的行驶时间明显大于IDM_LCA场景。同样, 因 为新生成的车辆必须等最接近道路入口的车辆行驶距离 大于最小安全距离才会驶入场景, 所以车流密度大于 10 以后, 车辆在两种场景下所需的行驶时间趋于一个定值。

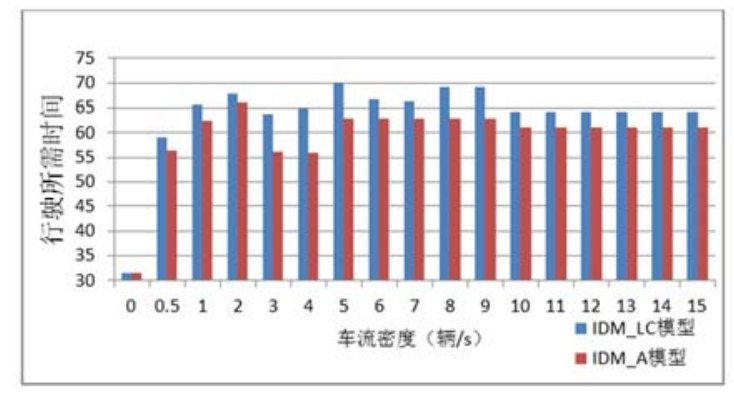

图9 紧急车辆的行驶1000所需时间。

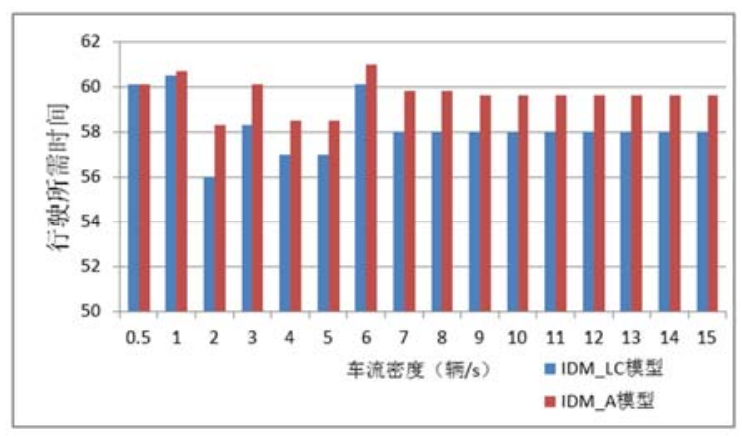

图10 车辆Vehicle ID=10行驶时间对比。
在VANET车载网络中, 有些路由算法对下一跳节点的 选择是在所有邻居节点中选取距离目标节点最近的点。这 种算法 (例如GPSR算法 [13]) 有时候会使数据被传输到一 个没有比目前节点更接近目的点的节点, 该节点的前向区 域就是路由空洞 [14]。路由空洞会对此类算法的路由协议 性能造成严重的影响, 导致数据无法正常传输, 使数据丢 包率上升。图 11 是不同车流密度下模拟时间为在 $20 \mathrm{~s}$ 和 $21 \mathrm{~s}$ 时, 紧急车辆与其同车道的车辆最近距离进行分析对比图。 显然, 在避让场景环境中, 中间车道上的车辆因受避让信 息的影响进行改道, 紧急车辆前面很长一段路都没有阻碍。 若是当前节点和目的节点都在中间车道方向, 在车流密度 低的环境下，是极易造成路由空洞的。

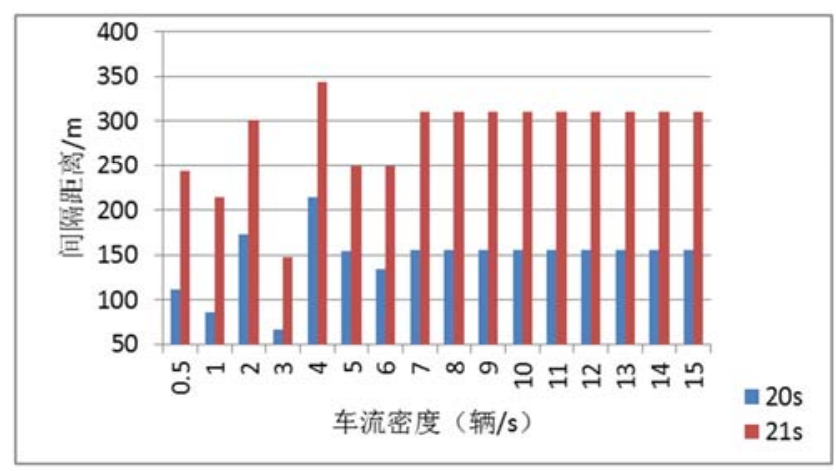

图11 紧急车辆空旷路段长度对比。

因为拥堵状态环境下, 无论急救车还是同路段的其他 车辆都很难在短时间内快速的改变行驶轨迹。所以以上分 析皆是基于路段没有堵塞状态。如果要模拟拥堵环境, 可 以在路段中设置障碍物。障碍物用静止的车辆模拟。生成 obstacle的方法是创建该类的对象然后调用Highway类中 的添加车辆的函数, 设置车辆的速度加速度以及方向为 0.0 。

\section{5. 结束}

本文在考虑真实的车辆运行特性以及驾驶员跟驰行 为的基础上引入对特殊车辆 (比如: 急救车、消防车) 的 避让行为, 对车载网仿真移动模型IDM_LC进行拓展, 提出 了一个考虑避让行为的移动模型IDM_A。并在网络模拟器 NS-3中实现了对避让移动模型的搭建。并在不同的场景下 对其进行模拟测试, 实验结果表明所提出的模型的可行性 和有效性。由于受到客观因素的限制, 本文所设定的模型 和场景环境与真实场景还存在较大的差距, 在今后的研究 工作中, 将对其进一步细化考虑。

\section{致谢}

本文为国家自然基金项目《基于组关联的车载网络协 同通信关键技术研究》（61272485）的阶段性成果之一。 


\section{参考文献}

[1] Hassanabadi B, Shea C, Zhang L, et al. Clustering in vehicular ad hoc networks using affinity propagation [J]. Ad Hoc Networks, 2014, 13: 535-548.

[2] Kotz, D. , Newport, C. , Gray, R. S., Liu, J., Yuan, Y. \& Elliott, C. (2004). Experimental evaluation of wireless simulation assumptions, Proc. ACM MSWiM' 04, ACM, New York, NY, USA, pp. 78-82.

[3] Vinícius F. S. Mota. Protocols, Felipe D. Cunha, Daniel F. Macedo, José M. S. Nogueira, Antonio A. F. Loureiro. mobility models and tools in opportunistic networks: A survey[J]. Computer Communications. 2014(48) :5-19.

[4] 魏达, 王沿锡, 王健, 刘衍街, 邓伟文. 车载自组网移动模 型综述 [J]. 计算机学报, 2013, 36 (4):677-700。

[5] Hannes Hartenstein, Kenneth P. Laberteaux. VANET车载 网技术及应用 $[\mathrm{M}]$. 孙立民，何云华，周新运，刘鸿亮，赵 茂华, 译. 北京: 清华大学出版社, 2013:93-131。

[6] Multiple Simulator Interlinking Environment for CC2C in VANETs, http://www. cn. uniduesseldorf. de/projects/MS IE, 2008.

[7] Simulation of Urban Mobility, http://sumo. sourceforg-ge. net

[8] The network simulator NS-2, http://www. isi. edu/nsnam/ns

[9] The $\mathrm{ns}^{-3}$ network simulator. [DB/OL]. http://www. nsnam. org , 2015

[10] NS-3 Manual. [DB/OL]. http://www. nsnam. org/docs/release/3. 22/manual/sing lehtml/ , 2014

[11] 肖书成, 邱珊. Ad hoc 网络及其路由性能研究 [J]. 重庆邮 电学院报（自然科学版），2004，16（3）：103-107。

[12] Bradley Dupont. Improvements in VANET Simulator in NS-3[D]. Virginia: Department of Computer Science 01d Dominion University, 2011.

[13] KARP B, KUNG H T. GPSR: greedy perimeler stateless routing for wireless network[C]//Pro of ACM/IEEE International conference on Mobile Computing and Networking. New York: ACM press, 2000: 243-254

[14] Fang Qing, Gao Jie, Guibas L. Locating and Bypassing Routing Holes in Sensor Networks[c]//Proc. of the 23rd Annual Joint Conference on IEEE Computer cmmunicatons Societies. New York, USA: Springer Netherlands, 2004:2458-2468. 